\title{
An ATP Model for Photovoltaic Module Considering the Temperature Dependence
}

\author{
Yaw-Juen Wang ${ }^{+}$, Yu-Sheng Juan and Chun-Han Wu \\ E. E. Dept., National Yunlin University of Science and Technology, Taiwan, ROC
}

\begin{abstract}
ATP (Alternative Transients Program) is a widely used simulation program for the electric power network. It provides the Shockley diode component that allows the user to quickly build a photovoltaic (PV) module model that can be used for simulation of an electric grid containing solar power generators. However, the diode parameters are inputted through a dialog window and cannot be varied during the simulation, which limits the program's performance of simulating cases where the PV module temperature undergoes variations. This paper proposes a method to solve this shortcoming by inserting a temperature-compensated current source in the existing equivalent circuit of PV module. Results obtained by the proposed model and by the analytical calculation are compared for validation.
\end{abstract}

Keywords: ATP, compensation, photovoltaic, shockley diode, temperature.

\section{Introduction}

Photovoltaic (PV) power has become an economically competitive renewable energy source in tropical and subtropical countries where abundant sunshine is available. Modeling of PV module plays an important role in the planning, operation and performance prediction of an electric grid with high level of PV capacity. The nonlinear current-voltage $(I-V)$ relation of PV module is sensitive to solar irradiance and temperature, which makes modeling of PV module challenging. PV module models can roughly be divided into two categories: equation-based and circuit-based models. The former describes the $I-V$ relation using a set of linear and nonlinear equations that are less intuitive to understand and apply. On the other hand, the circuitbased model uses an equivalent circuit to emulate the $I-V$ characteristics of a real PV module. Since electrical engineers are familiar with solving electric circuits, the circuit-based models have been overwhelmingly used in the literature. Fig. 1(a) shows the commonly used single-diode equivalent circuit of PV module.

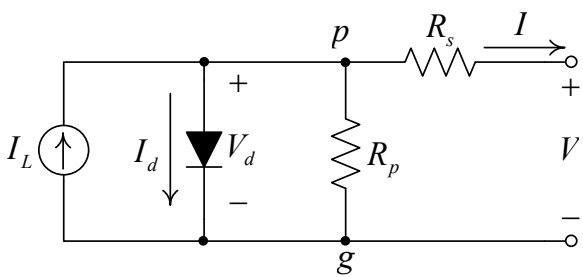

(a)

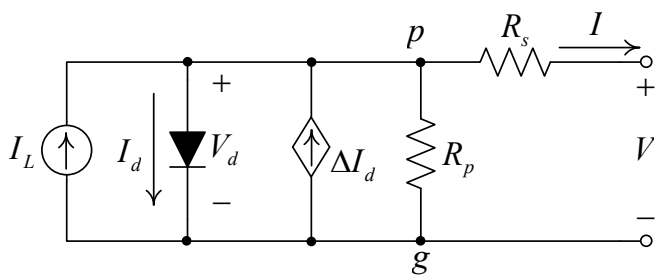

(b)

Fig. 1: Equivalent circuit of a PV module: (a) basic model, (b) proposed model containing a compensation current $\Delta I_{d}$.

The nonlinearity of the $I-V$ relation of PV module mainly comes from the diode. To build a PV module model in a circuit simulation program, the diode can be approximated by piecewise linear circuits. For example, Wang and Hsu [1] used several piecewise linear parallel branches (PLPB) to model a PV module.

\footnotetext{
Corresponding author. Tel.: + 886-5-5342601; fax: +886-5-5312065.

E-mail address: wangyj@yuntech.edu.tw.
} 
The piecewise linear approximation method was also used in Wang and Sheu's work [2] to construct the PLSB (piecewise linear series blocks) model. Both the PLPB and PLSB models are applicable to ATP (Alternative Transients Program), which is a power system simulation program widely used in many universities and power companies around the world. Starting from 2006, ATPDraw [3], the graphical preprocessor of ATP, has supported the Shockley diode component, which allows the user to simulate the nonlinear $I-V$ relation in ATP. This Shockley diode component was then used in Wang, Huang and Zeng's work [4] to model PV cells and modules for studying partially shadowed PV arrays. The PV module model of Ref. [4] is much easier to build than the PLPB and PLSB models, but it has a shortcoming of being only able to simulate PV modules at a fixed temperature since the temperature-related parameters of the Shockley diode model are manually keyed into a dialog window each time before a simulation run. To overcome this limitation, Wang and Huang [5] introduced a temperature-compensated voltage to work with a fixedparameter Shockley diode to consider the temperature effect on the $I-V$ characteristics of the PV module. Although the temperature-compensated voltage allows the temperature effects to be taken into account, the accuracy of the $I-V$ curves simulated by that model is not satisfactory. In this paper, the authors propose a new idea of temperature-controlled current source which is connected in parallel with a Shockley diode with fixed parameters. The compensation current is controlled according to the temperature and the voltage across the Shockley diode. The curve-fitting method has been used to accurately calculate the required compensation current. The $I-V$ and $P-V$ (power-voltage) curves simulated by the proposed model will be compared with those obtained from the analytical model for validation.

\section{Modeling of PV Module}

Before we proceed to construct a circuit model of PV module with temperature-controlled current source, we briefly review the analytical model based on the circuit of Fig. 1(a). More detailed description of the analytical model can be found in [6].

\subsection{Analytical Model}

In Fig. 1(a), $I_{L}$ refers to the light-generated current that can be written as

$$
I_{L}=\left(\frac{G}{G_{0}}\right) I_{g 0}\left(1+\alpha\left(T_{c}-T_{r e f}\right)\right)
$$

where $G$ is the solar irradiance, $G_{0}$ the reference irradiance taking the value of $1.0 \mathrm{~kW} / \mathrm{m}^{2}, I_{\mathrm{g} 0}$ the lightgenerated current at $G_{0}$ and $T_{r e f}$, $\alpha$ the temperature coefficient of $I_{L}, T_{c}$ the PV cell temperature in ${ }^{\circ} \mathrm{C}, T_{r e f}$ the reference temperature usually taking the value of $25^{\circ} \mathrm{C}$. The diode current $I_{d}$ can be expressed by the Shockley equation

$$
I_{d}\left(V_{d}, T_{c}\right)=I_{0}\left(T_{c}\right) \cdot\left(\exp \left(\frac{q V_{d}}{n k\left(T_{c}+273\right)}\right)-1\right)
$$

where $V_{d}$ is the voltage across the diode, $I_{0}$ the reverse saturation current, which is a sensitive function of temperature, $q$ the electron charge, $n$ the ideality factor, $k$ the Boltzmann constant. Applying the Kirchhoff's current law at node $p$ allows the $I-V$ relation of PV module to be written as an implicit function $f\left(G, T_{c}\right)$ as

$$
f\left(G, T_{c}\right)=I_{L}\left(G, T_{c}\right)-I_{d}\left(V_{d}, T_{c}\right)-\frac{V+I R_{s}}{R_{p}}-I=0
$$

where $V$ is the module voltage, $I$ the module current, $R_{s}$ and $R_{p}$ the series and parallel resistances. The diode voltage is given by $V_{d}=V+I R_{s}$. An $I-V$ curve at a given temperature and irradiance level can be obtained by solving eq. (3). Since eq. (3) is nonlinear, a numerical method, e.g., Newton-Raphson method, needs to be adopted to find the solution.

\subsection{Temperature-Compensated Current}

Fig. 1(b) shows the proposed equivalent circuit model: a fixed-parameter Shockley diode compensated by a current $\Delta I_{d}$ that is a function of $V_{d}$ and $T_{c}$. Although the diode cannot respond to temperature because its parameters have been preset and fixed at a base temperature $T_{b}$, the compensation current can correct this error. The base temperature can be chosen arbitrarily, but a value around the midpoint of the operational temperature interval is recommended. The compensation current is defined as 


$$
\Delta I_{d}\left(V_{d}, T_{c}\right)=I_{d}\left(V_{d}, T_{b}\right)-I_{d}\left(V_{d}, T_{c}\right)
$$

At a given temperature, the curve of $\Delta I_{d}$ against $V_{d}$ can be approximated by an exponential function

$$
\Delta I_{d}\left(V_{d}, T_{c}\right) \cong \Delta I_{a p}\left(V_{d}, T_{c}\right)=A \cdot \exp \left(B \cdot V_{d}\right)+C
$$

where $A, B$, and $C$ are the parameters of the exponential function and can be found using the curve fitting technique. It is noted that coefficients $A, B$, and $C$ vary with temperature $T_{c}$, so they can be fitted by known functions. In this paper, the three coefficients will be fitted by polynomials. Finally, the diode current at a given voltage $V_{d}$ and a given temperature $T_{c}$ can be approximately given by

$$
I_{d}\left(V_{d}, T_{c}\right) \cong I_{d}\left(V_{d}, T_{b}\right)-\Delta I_{a p}\left(V_{d}, T_{c}\right)
$$

\section{Numerical Examples}

The SM50 PV module manufactured by Shi-lin Electric Co., Taiwan, is taken as an example to illustrate how to build the proposed circuit model for simulation on ATP. Table 1 lists the module's electrical specifications. The parameters of the equivalent circuit for this module are listed in Table 2.

\begin{tabular}{c|c} 
Table 1: SM50 PV module electrical specifications \\
\hline \hline Specification & Quantity \\
\hline Rated power & $50 \mathrm{~W}_{\mathrm{p}}$ \\
Current at maximum power point & $2.7 \mathrm{~A}$ \\
Voltage at maximum power point & $18 \mathrm{~V}$ \\
Average short-circuit current & $3.3 \mathrm{~A}$ \\
Average open-circuit voltage & $22 \mathrm{~V}$ \\
\hline \hline
\end{tabular}

Table 2: Parameters of SM50 PV module

\begin{tabular}{l|c}
\hline \multicolumn{1}{c|}{ Parameter } & Quantity \\
\hline Reverse saturation current at $T_{\text {ref }}\left(I_{0}\right)$ & $2 \times 10^{-7} \mathrm{~A}$ \\
Ideality factor $(n)$ & 1.51 \\
Series resistance $\left(R_{s}\right)$ & $1.116 \Omega$ \\
Parallel resistance $\left(R_{p}\right)$ & $540 \Omega$ \\
Light-generated current at reference irradiance $\left(I_{\mathrm{g} 0}\right)$ & $3.3 \mathrm{~A}$ \\
Reference value of irradiance $\left(G_{0}\right)$ & $1 \mathrm{~kW} / \mathrm{m}^{2}$ \\
Reference temperature $\left(T_{\text {ref }}\right)$ & $25^{\circ} \mathrm{C}$ \\
\hline \hline
\end{tabular}

\subsection{Curve Fitting for the Compensation Current}

With the data listed in Table 2, the diode $I-V$ curves at different temperatures can be calculated by eq. (2) and are traced in Fig. 2(a) in which the dashed curve (corresponding to the base temperature $T_{b}=56.25^{\circ} \mathrm{C}$ ) is taken as the base curve. In Fig. 2(b), the solid lines are the compensation current $\Delta I_{d}$ calculated by eq. (4), while the scattered dots are the results of curve fitting using the exponential function of eq. (5). The parameters $A, B$, and $C$ obtained by curve fitting for temperature $T_{c}$ ranging from -20 to $70^{\circ} \mathrm{C}$ with an increment of $10^{\circ} \mathrm{C}$, are depicted in scattered dots in Fig. 3

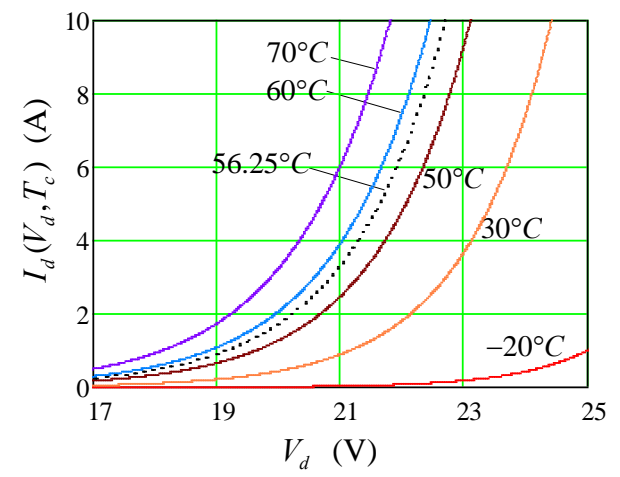

(a)

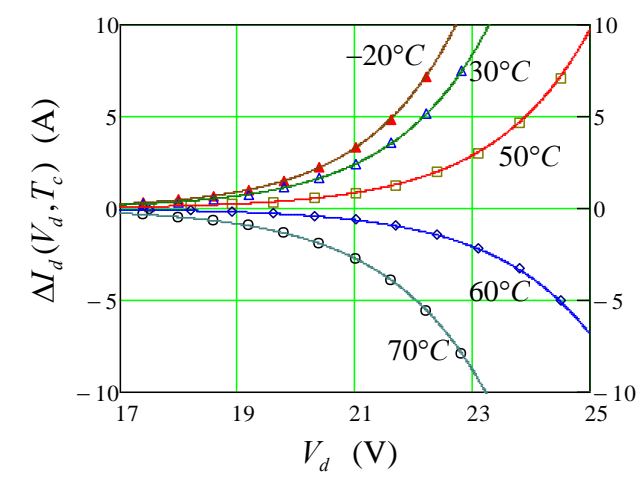

(b)

Fig. 2: (a) diode $I$ - $V$ curves at different temperatures, (b) $\Delta I_{d}$ as a function of $V_{d}$, parametered by $T_{c}$.

\subsection{Curve Fitting for $A\left(T_{c}\right), B\left(T_{c}\right)$, and $C\left(T_{c}\right)$}


The parameters $A, B$, and $C$ of the exponential function shown in eq. (5) vary with temperature $T_{c}$, hence we can make curve fitting for $A\left(T_{c}\right), B\left(T_{c}\right)$, and $C\left(T_{c}\right)$ with polynomials. We fit $A\left(T_{c}\right)$ with a 5th-order polynomial $A\left(T_{c}\right) \cong a_{0}+a_{1} T_{c}+a_{2} T_{c}^{2}+a_{3} T_{c}^{3}+a_{4} T_{c}^{4}+a_{5} T_{c}^{5}$. Likewise, $B\left(T_{c}\right)$ is fitted by a 2 nd-order polynomial $B\left(T_{c}\right) \cong b_{0}+b_{1} T_{c}+b_{2} T_{c}^{2}$ and $C\left(T_{c}\right)$ by a 4 th-order polynomial $C\left(T_{c}\right) \cong c_{0}+c_{1} T_{c}+c_{2} T_{c}^{2}+c_{3} T_{c}^{3}+$ $c_{4} T_{c}^{4}$. The polynomial coefficients are listed in Table 3. Fig. 3 shows that the three functions are well fitted with the polynomials.

Table 3. Polynomial coefficients for fitting $A\left(T_{c}\right), B\left(T_{c}\right)$, and $C\left(T_{c}\right)$

\begin{tabular}{l|l||l|l||l|l}
\hline \hline$a_{0}$ & $4.7280642 \times 10^{-6}$ & $b_{0}$ & 0.6395587 & $c_{0}$ & $-2.15136 \times 10^{-3}$ \\
$a_{1}$ & $2.66765 \times 10^{-8}$ & $b_{1}$ & $-3.9178477 \times 10^{-4}$ & $c_{1}$ & $-5.46716 \times 10^{-5}$ \\
$a_{2}$ & $-3.0819565 \times 10^{-10}$ & $b_{2}$ & $-5.4395118 \times 10^{-6}$ & $c_{2}$ & $2.782246 \times 10^{-7}$ \\
$a_{3}$ & $-1.1821109 \times 10^{-11}$ & & & $c_{3}$ & $2.329924 \times 10^{-8}$ \\
$a_{4}$ & $7.0614837 \times 10^{-13}$ & & & $c_{4}$ & $1.881533 \times 10^{-11}$ \\
$a_{5}$ & $-1.8148206 \times 10^{-14}$ & & & & \\
\hline \hline
\end{tabular}
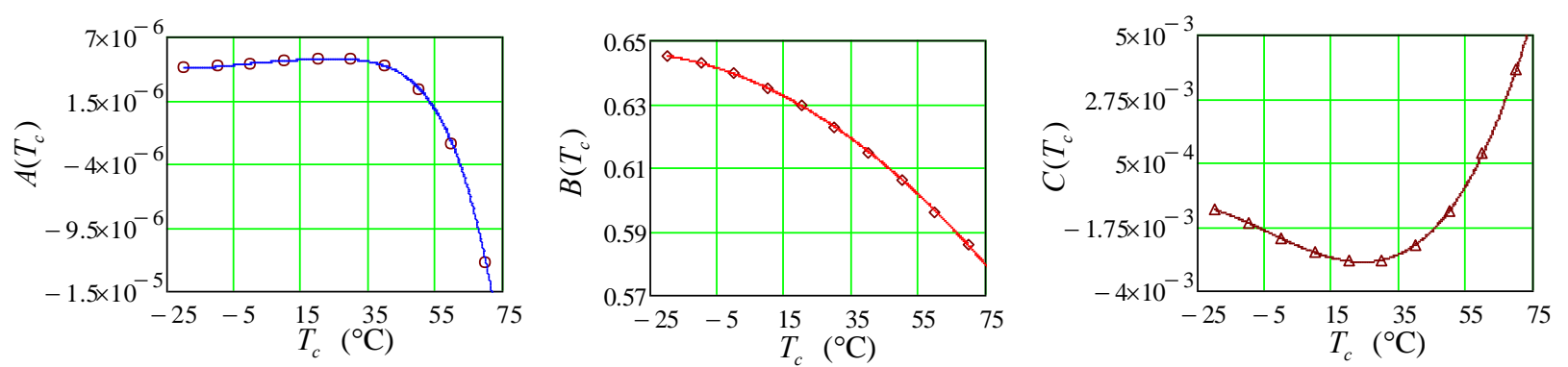

Fig. 3: Coefficients $A, B$, and $C$ of the exponential function eq. (5) fitted by polynomials.

\subsection{Model Built on ATP}

The proposed circuit model is built on ATP through the graphical pre-processor ATPDraw as shown in Fig. 3 which is comparable to Fig. 1(b) except that two controllers are introduced. In Fig. 3 the lightgenerated current $I_{L}$ is controlled by a Fortran statement block marked by $\mathrm{f}(\mathrm{u})$ that receives the irradiance $(G)$ and temperature $\left(T_{c}\right)$ signals and translates them into current signal $\left(I_{L}\right)$ as per eq. (1). The control of the temperature-compensated current $\Delta I_{d}$ is more complicated, and is accomplished by a MODELS [7] block that inputs the temperature $\left(T_{c}\right)$ and diode voltage $\left(v_{d}\right)$ signals and calculates $\Delta I_{d}$ as per the exponential function of eq. (5). Three temperature signals of $-5,35$, and $75^{\circ} \mathrm{C}$ are applied one after another in time. Connected to node $B$ is a sweeping voltage $v_{s}$ with saw-tooth wave. This arrangement allows the $I-V$ curves of the module at the three temperatures to be traced. Fig. 4(a) depicts the waveforms of the module output current $i$ and the sweeping voltage $v_{s}$ against the time axis. Notice that in this figure $v_{s}$ has been multiplied by a factor of 0.1 to reduce its amplitude. In this simulation run, the irradiance $G$ is set to be constantly equal to $1.0 \mathrm{~kW} / \mathrm{m}^{2}$ and the temperature $T_{c}$ evolves stepwise from $-5,35$, to $75^{\circ} \mathrm{C}$ in three seconds, so the current waveform readily shows the shape of $I-V$ characteristics. Fig. 4(b) shows the $I-V$ curves extracted from the ATP simulation (scattered dots) and calculated using the analytical model (solid lines). Good agreement has been achieved, showing the proposed model applicable and accurate for a module temperature ranging from -5 to $75^{\circ} \mathrm{C}$, largely covering the possible temperature changing range of a practical PV module.

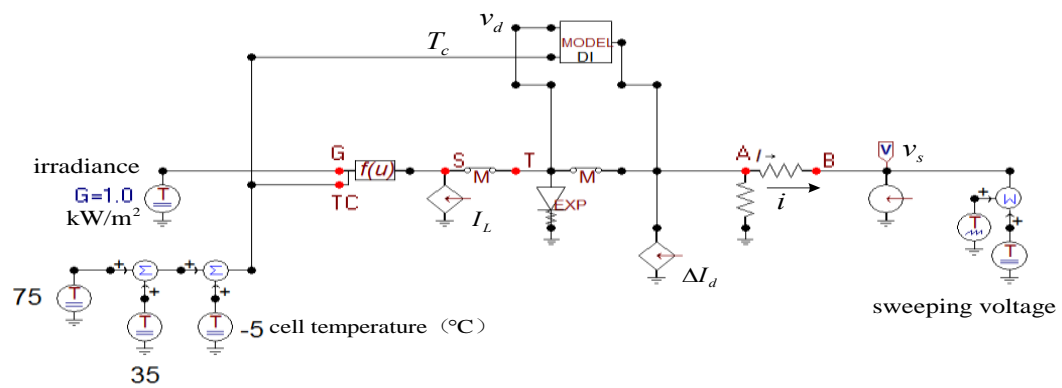

Fig. 3: Proposed circuit model realized and simulated on ATP. 


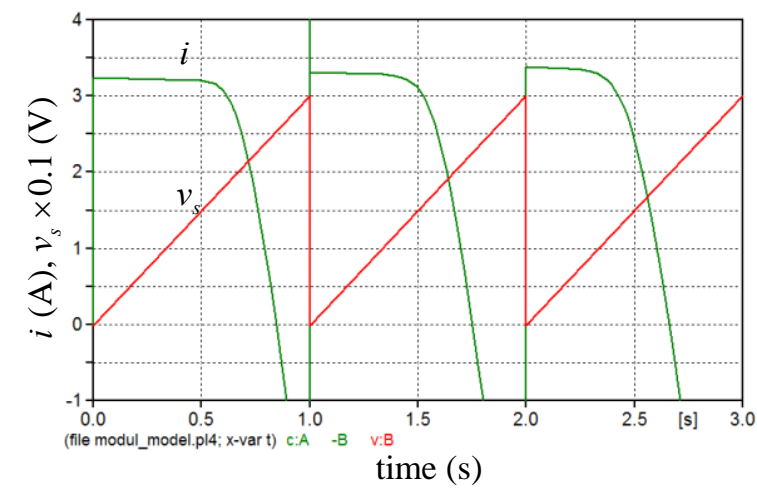

(a)

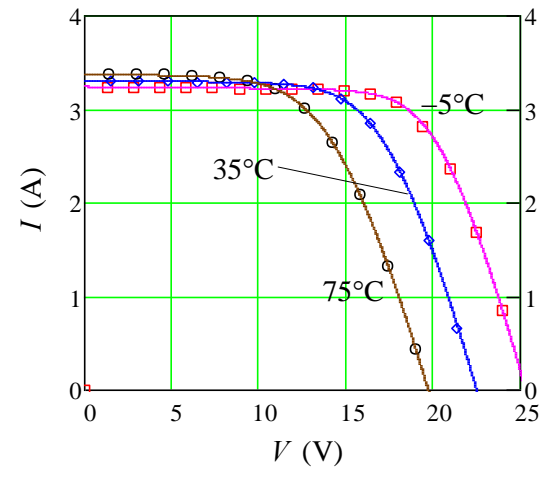

(b)

Fig. 4: (a) Waveforms of sweeping voltage $v_{s}$ (reduced by a factor of 0.1 ) and module current $i$ in time domain, (b) $I-V$ characteristics at $-5,35$ and $75^{\circ} \mathrm{C}$, obtained by the analytical model (solid lines) and ATP simulation (scattered dots).

\section{Conclusions}

A circuit model for PV module that constitutes a fixed-parameter Shockley diode component associated with a temperature-compensated current source has been proposed and presented to allow the model to be able to reflect the temperature effects on the $I-V$ characteristics of PV modules. Construction of the model involves two curve-fitting steps. In the first step, the compensation current is fitted to a three-parameter exponential function at several temperatures. This is followed in the second step by fitting the three parameters to three polynomials of appropriate orders to precisely express the variations of the parameters with the cell temperature. An example of modeling a 50-W practical PV module employing the proposed model has been carried out and tested on ATP for simulation over a cell temperature range from -5 to $75^{\circ} \mathrm{C}$. The simulation results are compared with those obtained from the analytical model. Good agreement between the proposed model and the analytical model has been achieved. Although the proposed model was tested on ATP, it is also applicable to other simulation programs for modeling PV systems. In this paper, we deliberately separate solar irradiance and cell temperature as two independent signals that can be controlled by the user. In most practical applications, cell temperature is closely correlated to solar irradiance and they can be combined together to simplify the simulation.

\section{Acknowledgements}

This work was supported by a research grant from the Ministry of Science and Technology of Taiwan, ROC. (Grant number: MOST 106-2221-E-224-033)

\section{References}

[1] Y. J. Wang, and P. C. Hsu. Modelling of solar cells and modules using piecewise linear parallel branches. IET Renewable Power Generation 2011, 5 (3): 215-222.

[2] Y. J. Wang, and R. L. Sheu. A new piecewise linear circuit model for photovoltaic cells and modules. International Journal of Electrical Engineering 2015, 22 (5): 181-188.

[3] László Prikler and Hans Kristian Høidalen, ATPDraw Users' Manual, Preliminary Release No. 1.0, 2009.

[4] Y. J. Wang, M. J. Huang, and W. C. Zeng. Modeling of partially shaded photovoltaic modules using the Shockley diode model in the environment of the Alternative Transients Program. Proc. of International Conference on Alternative Energy in Developing Countries and Emerging Economies, Bangkok, Thailand, 2013, pp. 269-275.

[5] Y. J. Wang, and P. S. Huang. An improved ATP PV cell model considering temperature effect. Proc. of 10th GMSARN International Conference on Smart Energy, Environment and Community Development in GMS, Phnom Penh, Cambodia, 2015.

[6] Y. J. Wang and P. C. Hsu. Analytical modeling of partial shading and different orientation of photovoltaic modules. IET Renewable Power Generation 2010, 4 (3): 272-282.

[7] L. Dubé and I. Bonfanti. MODELS: A new simulation tool in the EMTP. European Transactions on Electric Power 1992, 2 (1): 45-50. 